\title{
MULTIVALENT FUNCTIONS STAR-LIKE IN ONE DIRECTION
}

\author{
BY \\ TERUO TAKATSUKA
}

1. Introduction. Recently J. Bender [1] has obtained the following representation theorem for the multivalently star-like functions of the class $C(p, q)$ which is given in the following form:

THEOREM A. A function $f(z)$ is said to be a member of the class $C(p, q)$, where $p$ and $q$ are positive integers with $p \geqq q$, if and only if there is a positive number $\rho$ such that

$$
\begin{gathered}
\operatorname{Re} \frac{z f^{\prime}(z)}{f(z)}>0, \quad \rho<|z|<1, \\
\int_{0}^{2 \pi} \operatorname{Re}\left[\frac{z f^{\prime}(z)}{f(z)}\right] d \theta=2 \pi p, \quad z=r e^{i \theta}, \quad \rho<r<1,
\end{gathered}
$$

and

$$
f(z)=z^{q}+\sum_{n=q+1}^{\infty} a_{n} z^{n}, \quad|z|<1 .
$$

Let $f(z) \in C(p, q)$. Then

$$
f(z)=[\phi(z)]^{p} z^{q-p} \prod_{s=1}^{p-q}\left(1-\alpha_{s}^{-1} z\right)\left(1-\bar{\alpha}_{s} z\right),
$$

where $\phi(z) \in C(1,1), 0<\left|\alpha_{i}\right|<1$ and $f\left(\alpha_{i}\right)=0$ for $i=1,2, \cdots,(p-q)$.

Moreover he has proved an extremal theorem for the functions of the class $C(p, q)$.

The set of analytic functions star-like in one direction has been introduced by S. Ozaki [3] and M. S. Robertson [4]. T. Umezawa [8] has obtained a wider sufficient condition for a function star-like of order at most $p$ in one direction and for a function convex of order at most $p$ in one direction.

The purpose of this note is to extend T. Umezawa's theorem into the form of Theorem 1 by using an idea of S. Ozaki [3], and we shall generalize J. Bender's representation theorem in $\$ 4$ and his extremal theorem for the functions of the

Received by the editors April 11, 1964 and, in revised form, September 29, 1964. 
class $C(p, q)$ in $\S 5$. Moreover, we shall obtain a generalization of W. C. Royster's theorem [5].

2. Functions star-like of order $p$ in one direction.

DEFINITION 1. Let $f(z)$ be meromorphic for $|z| \leqq r$ and continuous and $f(z) \neq 0$ on $|z|=r$. Furthermore let $C$ be the image curve of $|z|=r$ under $f(z)$. If $C$ is cut by a straight line passing through the origin in $2 p$, and not more than $2 p$ points, $f(z)$ is said to be star-like of order $p$ in one direction.

Now let $C_{1}$ denote the part of $|z|=r$ on which $d \arg f(z)>0$ when $z$ moves on $|z|=r$ positively and put $\int_{C_{1}} d \arg z=x$, and let $C_{2}$ denote the part of $|z|=r$ on which $d \arg f(z) \leqq 0$ when $z$ moves on $|z|=r$ positively and hence $\int_{C_{2}} d \arg z=2 \pi-x$. Besides, we put

$$
y_{1}=\int_{C_{1}} d \arg f(z), \quad y_{2}=-\int_{C_{2}} d \arg f(z) .
$$

Let $N(\phi)$ be the number of the points of intersection of the straight line $\arg f(z)=\phi$ (started from the origin) with $C$. Then it is evident that the total variation of $\arg f(z)$ on $C$ is given by $\int_{0}^{\pi} N(\phi) d \phi$. If $C$ is star-like of order at least $(p+1)$ in one direction, then we have

$$
\int_{0}^{\pi} N(\phi) d \phi=\int_{C}|d \arg f(z)| \geqq 2 \pi(p+1) .
$$

Hence we have

$$
y_{1}+y_{2}=\int_{c}|d \arg f(z)| \geqq 2 \pi(p+1) .
$$

On the other hand, when $n(a)$ denotes the number of $a$-points of $f(z)$ in $|z|<r$, we obtain

$$
y_{1}-y_{2}=\int_{C} d \arg f(z)=2 \pi\{n(0)-n(\infty)\} .
$$

From (2.3) and (2.4), we have

$$
\begin{aligned}
& y_{1} \geqq\{p+1+n(0)-n(\infty)\} \pi, \\
& y_{2} \geqq\{p+1-n(0)+n(\infty)\} \pi .
\end{aligned}
$$

From (2.3), (2.5) and (2.6) we obtain the following theorem.

THEOREM 1. Let $f(z)$ be meromorphic for $|z| \leqq r$ and continuous and $f(z) \neq 0$ on $|z|=r$. If $f(z)$ satisfies on $|z|=r$ one of the following conditions

$$
y_{1}=\int_{C_{1}} d \arg f(z)<(A+B) \pi,
$$




$$
\begin{aligned}
y_{2} & =-\int_{C_{2}} d \arg f(z)<(A-B) \pi, \\
y_{1}+y_{2} & =\int_{C}|d \arg f(z)|<2 \pi A,
\end{aligned}
$$

where $A=p+1, B=n(0)-n(\infty)$, then $f(z)$ is star-like of order at most $p$ in one direction.

REMARK 1. (2.9) is T. Umezawa's theorem [8].

THEOREM 2. Under the same assumption as Theorem 1, if $f(z)$ satisfies on $|z|=r$ one of the following conditions

$$
\begin{aligned}
& \operatorname{Re} \frac{z f^{\prime}(z)}{f(z)}<\frac{A+B}{2}, \\
& \operatorname{Re} \frac{z f^{\prime}(z)}{f(z)}>-\frac{A-B}{2}, \\
&\left|\operatorname{Re} \frac{z f^{\prime}(z)}{f(z)}\right|<A,
\end{aligned}
$$

where $A=p+1, B=n(0)-n(\infty)$, then $f(z)$ is star-like of order at most $p$ in one direction.

Proof. When we notice that

$$
\frac{(A+B) \pi}{x} \geqq \frac{A+B}{2}, \quad-\frac{(A-B) \pi}{2 \pi-x} \leqq-\frac{A-B}{2},
$$

we obtain (1) and (2) in (2.10) from (2.7) and (2.8) respectively. Moreover, we obtain easily (3) of (2.10) from (2.9).

REMARK 2. The relation (3) of (2.10) is T. Umezawa's theorem [8].

THEOREM 3. Under the same assumption as Theorem 1, if $f(z)$ satisfies on $|z|=r$ the following inequality

$$
-\frac{\alpha(A-B)}{2 \alpha-(A+B)}<\operatorname{Re}\left[\frac{z f^{\prime}(z)}{f(z)}\right]<\alpha,
$$

where $A=p+1, B=n(0)-n(\infty), A+B>0, A-B>0$ and $2 \alpha$ is an arbitrary number not less than $A+B$, then $f(z)$ is star-like of order at most $p$ in one direction.

Proof. We apply here the method which has been used by T. Umezawa [8]. Since we have on $|z|=r$

$$
y_{1}-y_{2}=\int_{c} d \arg f(z)=2 \pi B,
$$


we obtain

$$
y_{1}+y_{2}=\int_{C}|d \arg f(z)|=2 y_{1}-2 \pi B .
$$

Since we have the hypothesis (2.11), we obtain

$$
\begin{aligned}
& y_{1}<\alpha x, \\
& y_{2}<\alpha(A-B)(2 \pi-x) /(2 \alpha-A-B) .
\end{aligned}
$$

Let us show that, under these circumstances, $y_{1}<(A+B) \pi$. Suppose that

$$
y_{1} \geqq(A+B) \pi,
$$

then we obtain $y_{2} \geqq(A-B) \pi$ from (2.12), and we have from (2.15)

$$
(A-B) \pi<\alpha(A-B)(2 \pi-x) /(2 \alpha-A-B) .
$$

Since we have $A-B>0$ and $2 \alpha>A+B$ from the hypothesis, we have

$$
(A+B) \pi>\alpha x \text {. }
$$

But on the other hand by (2.14) and (2.17), we obtain $y_{1}<(A+B) \pi$. This contradicts (2.16). Hence we have $y_{1}<(A+B) \pi$ on $|z|=r$. Therefore, by (2.7) $f(z)$ is star-like of order at most $p$ in one direction.

COROLlaRY 1. Under the same assumption as Theorem 1, if $f(z)$ satisfies on $|z|=r$ the following inequality

$$
\left|\operatorname{Re} \frac{z f^{\prime}(z)}{f(z)}-B\right|<A
$$

where $A=p+1, B=n(0)-n(\infty), A+B>0, A-B>0$, then $f(z)$ is starlike of order at most $p$ in one direction.

Proof. If we put $\alpha=A+B$ in (2.11), then we have Corollary 1 .

3. Functions convex of order $p$ in one direction.

Definition 2. Let $f(z)$ be meromorphic for $|z| \leqq r$ and continuous and $f^{\prime}(z) \neq 0$ on $|z|=r$. Furthermore let $C$ be the image curve of $|z|=r$. If every straight line parallel to a direction cuts $C$ in not more than $2 p$ points and there exists at least one such straight line which cuts $C$ in $2 p$ points, then $f(z)$ is said to be convex of order $p$ in one direction.

LEMMA 1. If $z f^{\prime}(z)$ is star-like of order $p$ in one direction, then (i) $f(z)$ is convex of order at most $p$ in one direction, (ii) $f(z)$ is at most $[p+n(\infty)]$-valent and at least $\operatorname{Max}[n(\infty)-p, 1]$-valent for $|z| \leqq r$.

We owe this lemma to M. S. Robertson [4] and T. Umezawa [8]. 
We can prove the below-mentioned Theorem 4, Theorem 5, Theorem 6 and Corollary 2 by using Lemma 1 corresponding to Theorem 1, Theorem 2, Theorem 3 and Corollary 1.

THEOREM 4. Let $f(z)$ be meromorphic for $|z| \leqq r$ and continuous and $f^{\prime}(z) \neq 0$ on $|z|=r$. Let $n^{*}(a)$ denote the number of a-points of $f^{\prime}(z)$ in $|z|<r$. Moreover, let $C_{1}$ denote newly the part of $|z|=r$ on which $d \arg d f(z)>0$ and let $C_{2}$ denote the part of $|z|=r$ on which $d \arg d f(z) \leqq 0$. If $f(z)$ satisfies on $|z|=r$ one of the following conditions

$$
\begin{gathered}
\int_{C_{1}} d \arg d f(z)<(D+E) \pi, \\
-\int_{C_{2}} d \arg d f(z)<(D-E) \pi, \\
\int_{C}|d \arg d f(z)|<2 \pi D,
\end{gathered}
$$

where $D=p+1-n(\infty), E=n^{*}(0)-n^{*}(\infty)+1$, then $f(z)$ is convex of order at most $p-n(\infty)$ in one direction for $|z| \leqq r$. Further $f(z)$ is at most p-valent and at least $\operatorname{Max}[2 n(\infty)-p, 1]$-valent for $|z| \leqq r$.

THEOREM 5. Under the same assumption as Theorem 4, if $f(z)$ satisfies on $|z|=r$ one of the following conditions

$$
\begin{gathered}
1+\operatorname{Re} \frac{z f^{\prime \prime}(z)}{f^{\prime}(z)}<\frac{D+E}{2}, \\
1+\operatorname{Re} \frac{z f^{\prime \prime}(z)}{f^{\prime}(z)}>-\frac{D-E}{2}, \\
\left|1+\operatorname{Re} \frac{z f^{\prime \prime}(z)}{f^{\prime}(z)}\right|<D,
\end{gathered}
$$

where $D=p+1-n(\infty), E=n^{*}(0)-n^{*}(\infty)+1$, then $f(z)$ is convex of order at most $p-n(\infty)$ in one direction for $|z| \leqq r$. Further $f(z)$ is at most p-valent and at least $\operatorname{Max}[2 n(\infty)-p, 1]$-valent for $|z| \leqq r$.

THEOREM 6. Under the same assumption as Theorem 4, if $f(z)$ satisfies on $|z|=r$ the following condition

$$
-\frac{\beta(D-E)}{2 \beta-D-E}<1+\operatorname{Re} \frac{z f^{\prime \prime}(z)}{F^{\prime}(z)}<\beta,
$$

where $D=p+1-n(\infty), E=n^{*}(0)-n^{*}(\infty)+1, D+E>0, D-E>0$ and $2 \beta$ is an arbitrary number not less than $D+E$, then $f(z)$ is convex of order at 
most $p-n(\infty)$ in one direction for $|z| \leqq r$. Further $f(z)$ is at most p-valent and at least $\operatorname{Max}[2 n(\infty)-p, 1]$-valent for $|z| \leqq r$.

COROLlaRY 2. Under the same assumption as Theorem 4, if $f(z)$ satisfies on $|z|=r$ the following inequality

$$
\left|1+\operatorname{Re} \frac{z f^{\prime \prime}(z)}{f^{\prime}(z)}-E\right|<D,
$$

where $D=p+1-n(\infty), E=n^{*}(0)-n^{*}(\infty)+1, D+E>0, D-E>0$.

REMARK 3. Author [6] has generalized the Theorem 4.

4. The generalization of $\mathbf{J}$. Bender's theorem.

Definition 3. A function $f(z)$ is said to be a member of the class $S(p, q)$, where $p$ and $q$ are positive integers with $p \geqq q$, if and only if $f(z)$ is meromorphic for $|z|<1$ and there is a positive member $\rho$ such that

$$
\begin{aligned}
& \operatorname{Re} \frac{z f^{\prime}(z)}{f(z)}>-\gamma, \quad 0 \leqq \gamma \leqq \frac{1}{2}, \quad \rho<|z|<1, \\
& \int_{0}^{2 \pi} d \arg f\left(r e^{i \theta}\right)=2 \pi p, \quad z=r e^{i \theta}, \quad \rho<|z|<1,
\end{aligned}
$$

and

$$
f(z)=z^{q}+\sum_{n=q+1}^{\infty} a_{n} z^{n}, \quad|z|<1 .
$$

Then, $f(z)$ is star-like of order at most $p$ in one direction from (2) of the Theorem 2.

$S_{1}(p, q)$ denotes the class of functions of the class $S(p, q)$ which satisfy (4.1), (4.2) and (4.3) on $|z|=1$.

THEOREM 7. Let $f(z) \in S_{1}(p, q)$. Suppose that in addition to the qth order zero at $z=0$, the function $f(z)$ has exactly s zeros, $\alpha_{1}, \alpha_{2}, \cdots, \alpha_{s}$ and $t$ poles, $\beta_{1}, \beta_{2}, \cdots, \beta_{t}$, such that $0<\left|\alpha_{i}\right|<1,0<\left|\beta_{j}\right|<1, i=1,2, \cdots, s, j=1,2, \cdots, t$. Finally let $q+s>t$ and $q+s-t=p$. Then

$$
F(z)=f(z) z^{p-q} \prod_{i=1}^{s}\left(1-\alpha_{i}^{-1} z\right)^{-1}\left(1-\bar{\alpha}_{i} z\right)^{-1} \prod_{j=1}^{t}\left(1-\beta_{j}^{-1} z\right)\left(1-\bar{\beta}_{j} z\right)
$$

is a member of the class $S_{1}(p, p)$.

Proof. By our hypothesis $F(z)$ is regular for $|z|<1$ and has no zero in $|z|<1$ except the $p$ th order zero at the origin. Moreover, 


$$
\begin{gathered}
\frac{z F^{\prime}(z)}{F(z)}=\frac{z f^{\prime}(z)}{f(z)}+\sum_{i=1}^{s} \frac{\alpha_{i}-\bar{\alpha}_{i} z^{2}}{\alpha_{i}+\bar{\alpha}_{i} z^{2}-\left(1+\left|\alpha_{i}\right|^{2}\right) z}-\sum_{j=1}^{t} \frac{\beta_{j}-\bar{\beta}_{j} z^{2}}{\beta_{j}+\bar{\beta}_{j} z^{2}-\left(1+\left|\beta_{j}\right|^{2}\right) z}, \\
\operatorname{Re} \frac{e^{i \theta} F^{\prime}\left(e^{i \theta}\right)}{F\left(e^{i \theta}\right)}=\operatorname{Re} \frac{e^{i \theta} f^{\prime}\left(e^{i \theta}\right)}{f\left(e^{i \theta}\right)} .
\end{gathered}
$$

Thus on $|z|=1$, we have

$$
\operatorname{Re} \frac{z F^{\prime}(z)}{F(z)}=\operatorname{Re} \frac{z f^{\prime}(z)}{f(z)}>-\gamma,
$$

and since $F(z)$ has $p$ zeros in $|z|<1$, at the origin,

$$
\int_{0}^{2 \pi} d \arg f(z)=2 p \pi, \quad z=e^{i \theta}
$$

Hence $F(z) \in S_{1}(p, p)$.

THeOReM 8. Let $F(z) \in S(p, p)$. Then

$$
\begin{gathered}
\frac{|z|^{p}}{(1+|z|)^{2 p+2 \gamma}} \leqq|F(z)| \leqq \frac{|z|^{p}}{(1-|z|)^{2 p+2 \gamma}}, \\
\left|\arg \frac{F(z)}{z^{p}}\right| \leqq(p+\gamma) \log \frac{1+|z|}{1-|z|} .
\end{gathered}
$$

To prove the above theorem we need the following lemma.

LEMMA 2. Let $F(z)$ be regular for $|z|<1$. If $\operatorname{Re} F(z) \geqq 0$ for $|z|<1$ and $F(0)=1$, then

$$
\left|F(z)-\frac{1+r^{2}}{1-r^{2}}\right|<\frac{2 r}{1-r^{2}} \quad \text { for } z=r e^{i \theta} \quad(0 \leqq r<1) .
$$

We owe this lemma to $Z$. Nehari [2, p. 173].

Proof of Theorem 8. Since $F(z)$ is regular for $|z| \leqq r$ and has $p$ zeros in $|z|<r$, at the origin, and satisfies

$$
\operatorname{Re}\left[\frac{z F^{\prime}(z)}{F(z)}+\gamma\right]>0
$$

we have by the Lemma 2

$$
\left|\frac{z F^{\prime}(z)}{F(z)}+\gamma-\frac{(p+\gamma)\left(1+|z|^{2}\right)}{1-|z|^{2}}\right| \leqq \frac{2(p+\gamma)|z|}{1-|z|^{2}} .
$$

Then we obtain 


$$
\left|\frac{z F^{\prime}(z)}{F(z)}-\frac{p}{z}-\frac{2(p+\gamma) \bar{z}}{1-|z|^{2}}\right| \leqq \frac{2(p+\gamma)}{1-|z|^{2}} .
$$

When we integrate along the radius $0 z$, we have by this relation

$$
\begin{aligned}
\mid \log \frac{F(z)}{z} & -(p+\gamma) \log \frac{1}{1-|z|^{2}} \mid \\
& \leqq \\
& \leqq \int_{0}^{z}\left\{\frac{F^{\prime}(z)}{F(z)}-\frac{p}{z}-\frac{2(p+\gamma) \bar{z}}{1-|z|^{2}}\right\} d z \mid \\
& \int_{0}^{|z|} \frac{2(p+\gamma)}{1-|z|^{2}} d|z|=(p+\gamma) \log \frac{1+|z|}{1-|z|}
\end{aligned}
$$

where we put the branch of $\log \left(F(z) / z^{p}\right)$ that makes $\log \left(F(z) / z^{p}\right)$ zero at the origin. Therefore, since $\log \left(F(z) / z^{p}\right)$ is in the circle whose center is

$$
-(p+\gamma) \log \left(1-|z|^{2}\right)
$$

and its radius is $(p+\gamma)\{\log (1+|z|)-\log (1-|z|)\}$, we obtain (4.5). When we put the imaginary part of $\log \left(F(z) / z^{p}\right)$ in (4.7), we obtain (4.6).

Theorem 9 (The Representation TheOrem). Let $f(z) \in S(p, q)$. Suppose that $f(z)$ has exactly $s$ zeros, $\alpha_{1}, \alpha_{2}, \cdots, \alpha_{s}$ and $t$ poles $\beta_{1}, \beta_{2}, \cdots, \beta_{t}$, such that $0<\left|\alpha_{i}\right|<1,0<\left|\beta_{j}\right|<1, i=1,2, \cdots, s, j=1,2, \cdots, t$. Let $q+s>$ tand $q+s-t=p$. Then we have

$$
f(z)=F(z) z^{q-p} \prod_{i=1}^{s}\left(1-\alpha_{i}^{-1} z\right)\left(1-\bar{\alpha}_{i} z\right) \prod_{j=1}^{t}\left(1-\beta_{j}^{-1} z\right)^{-1}\left(1-\bar{\beta}_{j} z\right)^{-1},
$$

where $F(z) \in S(p, p)$.

Proof. Consider the sequence $\left\{G_{n}(z)\right\}$, where $G_{n}(z)=f\left(t_{n} z\right)$ when $\rho^{-1}>t_{n}>1$ and $\lim _{n \rightarrow \infty} t_{n}=1$. Then, with $z=r e^{i \theta}, G_{n}(z)$ satisfies (4.1) and (4.2) when $\rho t_{n}^{-1}<r<t_{n}^{-1}$. Thus for each $n, G(z)_{n} \in S_{1}(p, q)$. Applying Theorem 7, we have

$$
F_{n}(z)=G_{n}(z) z^{p-q} \prod_{i=1}^{s}\left(1-\alpha_{i}^{-1} t_{n} z\right)^{-1}\left(1-z t_{n} \bar{\alpha}_{i}\right)^{-1} \prod_{j=1}^{t}\left(1-\beta_{j}^{-1} t_{n} z\right)\left(1-\bar{\beta}_{j} t_{n} z\right),
$$

where $F_{n}(z) \in S_{1}(p, p)$. Since $F_{n}(z)$ is regular for $|z|<1$ and has no zero in $|z|<1$ except the $p$ th order zero at the origin, we have $\lim _{n \rightarrow \infty} F_{n}(z)=F(z)$ uniformly in every closed subdomain interior to $|z|<1$. Then we obtain (4.8).

Definition 4. A function $f(z)$ is said to be a member of the class $K(p, q)$ if and only if $f(z)$ has the representation given by equation (4.8).

\section{An extremal theorem for the class $K(p, q)$.}

THEOREM 10. Let $f(z) \in K(p, q)$. Then 


$$
\begin{aligned}
\left|a_{n}\right| & \leqq\left|A_{n}\right|, \quad n=q+1, q+2, \cdots, \\
\left|f\left(r e^{i \theta}\right)\right| & \leqq H(r), \quad z=r e^{i \theta},
\end{aligned}
$$

where $A_{n}$ and $H(z)$ are defined by

$$
\begin{aligned}
H(z)= & \frac{z^{q}}{(1-z)^{2 p+2 \gamma}} \prod_{i=1}^{s}\left(1-\left|\alpha_{i}\right|^{-1} z\right)\left(1-\left|\alpha_{i}\right| z\right) \\
& \cdot \prod_{j=1}^{t}\left(1-\left|\beta_{j}\right|^{-1} z\right)^{-1}\left(1-\left|\beta_{j}\right| z\right)^{-1} \\
= & z^{q}+\sum_{n=q+1}^{\infty} A_{n} z^{n} \text { for }|z| \operatorname{Min}\left[\left|\beta_{j}\right|, j=1,2, \cdots, t\right] .
\end{aligned}
$$

Proof. By Theorem 9 we have

$$
\begin{aligned}
f(z) & =z^{q}+\sum_{n=q+1}^{\infty} a_{n} z^{n} \\
& =F(z) z^{q-p} \prod_{i=1}^{s}\left(1-\alpha_{i}^{-1} z\right)\left(1-\bar{\alpha}_{i} z\right) \prod_{j=1}^{t}\left(1-\beta_{j}^{-1} z\right)^{-1}\left(1-\bar{\beta}_{j} z\right)^{-1} .
\end{aligned}
$$

Since $F(z)$ is regular for $|z|<r$ and $F(z) \neq 0$ or $\infty$ for $0<|z|<r$ and $\operatorname{Re}\left\{z F^{\prime}(z) / F(z)+\gamma\right\}>0$, we have by Carathéodory's theorem

$$
\left\{z F^{\prime}(z) / F(z)+\gamma\right\} \ll(p+\gamma)(1+z) /(1-z) .
$$

Therefore we have

$$
F(z) \ll z^{q} /(1-z)^{2 p+2 \gamma} .
$$

We have also for $|z|<\operatorname{Min}\left[\left|\beta_{j}\right|, j=1,2, \cdots, t\right]$

$$
\prod_{j=1}^{t}\left(1-\beta_{j}^{-1} z\right)^{-1}\left(1-\bar{\beta}_{j} z\right)^{-1} \ll \prod_{j=1}^{t}\left(1-\left|\beta_{j}\right|^{-1} z\right)^{-1}\left(1-\left|\beta_{j}\right| z\right)^{-1} .
$$

Then, by using (5.4), (5.5) and (5.6) we obtain (5.1). Therefore, the bound (5.2) follows readily from (5.1).

THEOREM 11. Let $f(z) \in K(p, q)$. Then

$$
\begin{aligned}
\left|f\left(r e^{i \theta}\right)\right| \geqq & \frac{r^{q}}{(1+r)^{2 p+2 \gamma}} \prod_{i=1}^{s}\left(1-\frac{r}{\left|\alpha_{i}\right|}\right)\left(1-\left|\alpha_{i}\right| r\right) \\
& \cdot \prod_{j=1}^{t}\left(1+\frac{r}{\left|\beta_{j}\right|}\right)^{-1}\left(1+\left|\beta_{j}\right| r\right)^{-1}
\end{aligned}
$$

for $r<\operatorname{Min}\left[\left|\beta_{j}\right|, j=1,2, \cdots, t\right]$.

Proof. The bound (5.7) follows immediately from Theorem 8 and Theorem 9. 
THEOREM 12. Let $f(z)=z^{-p}+a_{-p+1} z^{-p+1}+\cdots$ be regular except for a pole of order $p$ at the origin in $|z|<1$. If $f(z)$ satisfies the following conditions

$$
\begin{gathered}
\operatorname{Re}\left[\frac{z f^{\prime}(z)}{f(z)}\right]<\varepsilon, \quad 0 \leqq \varepsilon \leqq \frac{1}{2}, \quad 0<|z| \leqq r<1, \\
\int_{0}^{2 \pi} \operatorname{Re}\left[\frac{z f^{\prime}(z)}{f(z)}\right] d \theta=-2 p \pi, \quad z=r e^{i \theta}, \quad 0<|z| \leqq r<1,
\end{gathered}
$$

then $f(z)$ is star-like of order at most $p$ in one direction and we have

$$
\begin{aligned}
\frac{(1-|z|)^{2 p+2 \varepsilon}}{|z|^{p}} & \leqq|f(z)| \leqq \frac{(1+|z|)^{2 p+2 \varepsilon}}{|z|^{p}} \\
\left|a_{-p+k}\right| & \leqq \frac{(2 p+2 \varepsilon)(2 p+2 \varepsilon-1) \cdots(2 p+2 \varepsilon-k+1)}{k !}
\end{aligned}
$$

for $k=1,2, \cdots, p$, and

$$
\left|\arg z^{p} f(z)\right| \leqq(p+\varepsilon) \log \frac{1+|z|}{1-|z|} .
$$

Proof. $f(z)$ is star-like of order at most $p$ in one direction from (1) of the Theorem 2. Since we have

$$
\frac{z f^{\prime}(z)}{f(z)}-\varepsilon=-p-\varepsilon+\sum_{n=1}^{\infty} b_{n} z^{n}
$$

we obtain from the assumption

$$
\operatorname{Re}\left[\frac{z f^{\prime}(z)}{f(z)}-\varepsilon\right] /(-p-\varepsilon)>0 .
$$

Then we obtain from Lemma 2

$$
\left|\frac{z f^{\prime}(z)}{f(z)}+p+\frac{2(p+\varepsilon)|z|^{2}}{1-|z|}\right| \leqq \frac{2(p+\varepsilon)|z|}{1-|z|^{2}} .
$$

When we integrate along the radius $0 z$, we have

$$
\begin{aligned}
\mid \log z^{p} f(z) & -(p+\varepsilon) \log \left(1-|z|^{2}\right) \mid \\
& \leqq\left|\int_{0}^{z}\left(\frac{f^{\prime}(z)}{f(z)}+\frac{p}{z}-\frac{2(p+\varepsilon) \bar{z}}{1-|z|^{2}}\right) d z\right| \\
& \leqq \int_{0}^{|z|} \frac{2(p+\varepsilon)}{1-|z|^{2}} d|z|=(p+\varepsilon) \log \frac{1+|z|}{1-|z|}
\end{aligned}
$$

where we put the branch of $\log \left(z^{p} f(z)\right)$ that makes $\log \left(z^{p} f(z)\right)$ zero at the origin. 
Therefore, since $\log \left(z^{p} f(z)\right)$ is in the circle whose center is $(p+\varepsilon) \log \left(1-|z|^{2}\right)$ and its radius is $(p+\varepsilon)\{\log (1+|z|)-\log (1-|z|)\}$, we obtain (5.10). When we put the imaginary part of $\log \left(z^{p} f(z)\right)$ in (5.13), we obtain (5.12). Moreover, since we have $\operatorname{Re}\left\{z f^{\prime}(z) / f(z)-\varepsilon\right\}<0$, we obtain by Carathéodory's theorem $f(z) \ll(1+z)^{2 p+2 \varepsilon} / z^{p}$. Therefore, from this relation we obtain (5.11).

REMARK 4. Theorem 9, Theorem 10 and Theorem 11 are generalizations of J. Bender's theorem [1]. Further, when we put $\varepsilon=0$ in (5.11) of Theorem 12, we obtain W. C. Royster's theorem [5]. Moreover, if $F(z)=z^{p}+a_{p+1} z^{p+1}+\cdots$ is regular and star-like of order $p$ in the direction of one diametral line of $F(z)$ for $|z| \leqq 1$, then there exists the relation

$$
\left|a_{p+n}\right| \leqq\left|\left(\begin{array}{c}
-2 p \\
n
\end{array}\right)\right|
$$

This relation was proved in [7].

I wish to express here my hearty gratitude to Professor S. Ozaki for his kind guidance during my research.

\section{REFERENCES}

1. J. Bender, Some extremal theorems for multivalently star-like function, Duke Math. J. 29 (1962), 101-106.

2. Z. Nehari, Conformal mapping, McGraw-Hill, New York, 1952.

3. S. Ozaki, On the theory of multivalent functions. II, Sci. Rep. Tokyo Bunrika Daigaku A 4 (1941), 45-87.

4. M. S. Robertson, Analytic functions star-like in one direction, Amer. J. Math. 58 (1936), 465-472.

5. W. C. Royster, Meromorphic starlike multivalent functions, Trans. Amer. Math. Soc. 107 (1963), 300-308.

6. T. Takatsuka, On the theory of multivalent functions, Bull. Ed. Fac. Shizuoka Univ. Japan 14 (1963), 26-33.

7. T. Takatsuka, T. Umezawa and S. Ozaki, Analytic functions star-like of order $p$ in one direction, Bull. Ed. Fac. Shizuoka Univ. Japan 1 (1950), 81-87.

8. T. Umezawa, Analytic functions convex in one direction, J. Math. Sci. Japan 4 (1952), 194-202.

SHIZUOKA UNIVERSITY,

SHIZUOKA, JAPAN 